\title{
On the self-constraint mechanism of the cross-stream secondary flow in a streamwise-rotating channel
}

Cite as: Phys. Fluids 32, 105115 (2020); https://doi.org/10.1063/5.0023695

Submitted: 31 July 2020 . Accepted: 28 September 2020 . Published Online: 09 October 2020

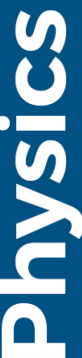

(D) Z. Yang (杨子轩), (D) B.-Q. Deng (邓冰清), (D) B.-C. Wang, and (D) L. Shen
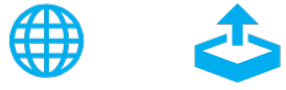

\section{ARTICLES YOU MAY BE INTERESTED IN}

Direct numerical simulation on the effects of surface slope and skewness on rough-wall turbulence

Physics of Fluids 32, 105113 (2020); https://doi.org/10.1063/5.0024038

On the wake flow behind a sphere in a pipe flow at low Reynolds numbers

Physics of Fluids 32, 103605 (2020); https://doi.org/10.1063/5.0017349

Large scale structures of turbulent flows in the atmospheric surface layer with and without sand

Physics of Fluids 32, 106604 (2020); https://doi.org/10.1063/5.0023126

\section{Physics of Fluids GALLERY OF GOVERS}

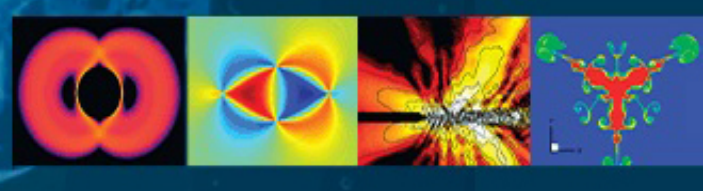




\title{
On the self-constraint mechanism of the cross-stream secondary flow in a streamwise-rotating channel (ip)
}

\author{
Cite as: Phys. Fluids 32, 105115 (2020); doi: 10.1063/5.0023695 \\ Submitted: 31 July 2020 - Accepted: 28 September 2020 • \\ Published Online: 9 October 2020
}

\author{
Z. Yang (杨子轩), ${ }^{1,2}$ (D) B.-Q. Deng (邓冰清), ${ }^{3, a)}$ (D) B.-C. Wang, ${ }^{4}$ (D) and L. Shen ${ }^{3}$ (D)
}

\begin{abstract}
AFFILIATIONS
${ }^{1}$ State Key Laboratory of Nonlinear Mechanics, Institute of Mechanics, Chinese Academy of Sciences, Beijing 100190, China

${ }^{2}$ School of Engineering Sciences, University of Chinese Academy of Sciences, Beijing 100190, China

${ }^{3}$ Department of Mechanical Engineering and St. Anthony Fall Laboratory, University of Minnesota, Minneapolis, Minnesota 55455, USA

${ }^{4}$ Department of Mechanical Engineering, University of Manitoba, Winnipeg, Manitoba R3T 5V6, Canada
\end{abstract}

\begin{abstract}
a) Author to whom correspondence should be addressed: bdeng@umn.edu
\end{abstract}

\begin{abstract}
The mechanism underlying the magnitude-reverse phenomenon of the mean spanwise velocity with respect to an increasing rotation number in a streamwise-rotating channel flow is investigated through a budget balance analysis of Reynolds shear stress $\left\langle u_{2}^{\prime} u_{3}^{\prime}\right\rangle$. It is found that $\left\langle u_{2}^{\prime} u_{3}^{\prime}\right\rangle$ imposes a negative feedback to itself through the production term, which prevents its magnitude from increasing monotonically with an increasing rotation number. This behavior of $\left\langle u_{2}^{\prime} u_{3}^{\prime}\right\rangle$ further leads to the magnitude reverse in the spanwise wall shear stress and mean spanwise velocity in the near-wall region.
\end{abstract}

Published under license by AIP Publishing. https://doi.org/10.1063/5.0023695

\section{INTRODUCTION}

Turbulent channel flows subjected to streamwise system rotation feature a distinct mean spanwise motion, of which the direction changes three times between the two walls of the channel, forming a "double S-shaped" profile ${ }^{1-9}$ (see Fig. 1). In laminar flows, the mean spanwise velocity is found to be the primary secondary flow from an instability analysis. ${ }^{10}$ In turbulent flows, the spanwise velocity is correlated with the Reynolds shear stress $\left\langle u_{2}^{\prime} u_{3}^{\prime}\right\rangle$, which is zero in non-rotating channel flows, but becomes non-trivial in streamwiserotating channel flows. In this paper, subscripts 1-3 denote the streamwise, wall-normal, and spanwise directions, respectively, the prime denotes the fluctuations, and a pair of angular brackets $\langle\cdot\rangle$ denote averaging over time and homogeneous directions, i.e., the $x_{1}$ and $x_{3}$-directions.

An important feature of the mean spanwise velocity $\left\langle u_{3}\right\rangle$ in the streamwise-rotating channel flow is the non-monotonic behavior of its magnitude with respect to an increasing rotation number., ${ }^{411}$ To be specific, at low rotation numbers, the magnitude of $\left\langle u_{3}\right\rangle$ increases with the rotation number. However, there exists a critical rotation number, above which the magnitude of $\left\langle u_{3}\right\rangle$ decreases as the rotation number continues to increase. Although such a reverse effect of the rotation number on the mean spanwise velocity is shown in many previous studies, the investigation of the underlying mechanism is limited.

The transport equations of Reynolds stresses, which reflect the contributions of different physical processes to the budget balances of turbulent kinetic energy and momentum fluxes, are useful for understanding the flow dynamics. ${ }^{12-16}$ For example, the sustaining mechanism of the large-scale roll cells in the streamwiserotating channel, namely, the Taylor-Görtler-like vortices, is elucidated through the investigation of the energy transport processes. ${ }^{17}$ In this paper, we explore the underlying mechanism of the nonmonotonic behavior of the mean spanwise velocity. The remainder of this paper is organized as follows. In Sec. II, the database used for the present study is described. In Sec. III, the non-monotonic behaviors of the magnitudes of $\left\langle u_{3}\right\rangle$ and $\left\langle u_{2}^{\prime} u_{3}^{\prime}\right\rangle$ with respect to the rotation number increase are demonstrated. The mechanism 


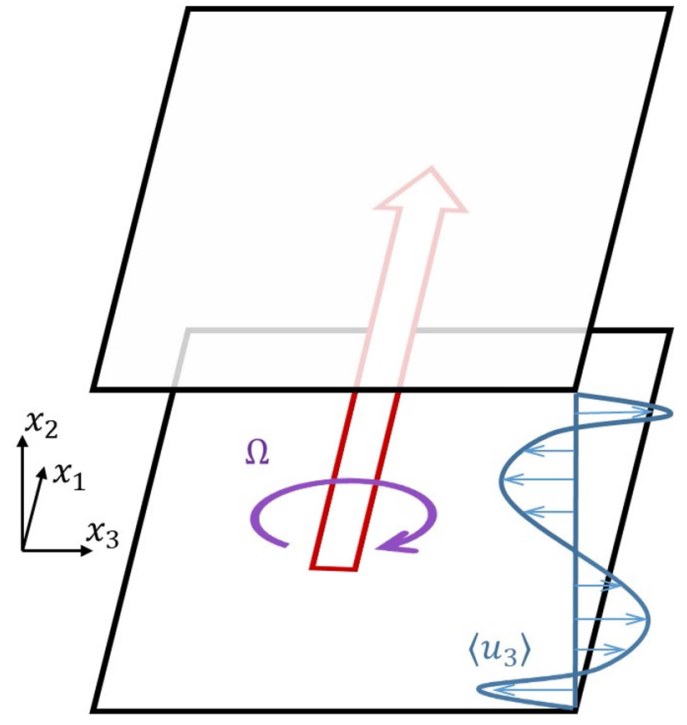

FIG. 1. Schematic of a turbulent channel flow subjected to a streamwise system rotation. The red hollow arrow points to the streamwise direction. The angular velocity of the system rotation is $\Omega$. The streamwise system rotation induces a mean spanwise secondary flow $\left\langle u_{3}\right\rangle$, which changes its direction three times between the two channel walls.

underlying the magnitude-reverse effect of the rotation number on $\left\langle u_{3}\right\rangle$ and $\left\langle u_{2}^{\prime} u_{3}^{\prime}\right\rangle$ is investigated through the analyses of the balance equation of mean spanwise shear stress and the transport equation of $\left\langle u_{2}^{\prime} u_{3}^{\prime}\right\rangle$ in Sec. IV, followed by a summary of major conclusions in Sec. V.

\section{DATABASE OF STREAMWISE-ROTATING CHANNEL FLOW}

The present study is conducted based on the database of streamwise-rotating channel flows established by Yang and Wang using direct numerical simulation (DNS). The governing equation of the DNS is expressed as

$$
\begin{gathered}
\frac{\partial u_{i}}{\partial x_{i}}=0 \\
\frac{\partial u_{i}}{\partial t}+u_{k} \frac{\partial u_{i}}{\partial x_{k}}=-\frac{1}{\rho} \frac{\partial p}{\partial x_{i}}+v \frac{\partial^{2} u_{i}}{\partial x_{k} \partial x_{k}}-2 \varepsilon_{i 1 k} \Omega u_{k}-\frac{\Pi}{\rho} \delta_{i 1},
\end{gathered}
$$

where $\rho$ and $v$ represent the density and kinematic viscosity of the fluid, respectively, $p$ is the pressure, $\Omega$ is the angular velocity of the streamwise system rotation, $\Pi$ is a constant mean streamwise pressure gradient that drives the flow, and $\varepsilon_{i j k}$ and $\delta_{i j}$ are Levi-Civita symbol and the Kronecker delta, respectively. A pseudo-spectral method code is utilized to solve Eqs. (1) and (2). A detailed description of the numerical algorithm is given in the work of Yang and Wang. ${ }^{18}$

Table I summarizes the key parameters of the DNS data. As shown, the rotation number $R o_{\tau}=2 \Omega h / u_{\tau}$ ranges from 0 to 150 , where $u_{\tau}=\sqrt{\Pi h / \rho}$ is the wall-friction velocity, while the Reynolds
TABLE I. Key parameters, computational domain, and grid resolution of DNS cases.

\begin{tabular}{lccccc}
\hline \hline$R o_{\tau}$ & $R o_{c}$ & $R e_{\tau}$ & $R e_{c}$ & $L_{1} \times L_{2} \times L_{3}$ & $N_{1} \times N_{2} \times N_{3}$ \\
\hline 0 & 0 & 180 & 3263 & $16 \pi h \times 2 h \times 8 \pi h$ & $512 \times 128 \times 512$ \\
7.5 & 0.44 & 180 & 3068 & $32 \pi h \times 2 h \times 8 \pi h$ & $1024 \times 128 \times 512$ \\
15 & 0.97 & 180 & 2784 & $64 \pi h \times 2 h \times 8 \pi h$ & $2048 \times 128 \times 512$ \\
30 & 2.17 & 180 & 2488 & $128 \pi h \times 2 h \times 8 \pi h$ & $4096 \times 128 \times 512$ \\
75 & 6.20 & 180 & 2177 & $256 \pi h \times 2 h \times 8 \pi h$ & $8192 \times 128 \times 512$ \\
150 & 12.92 & 180 & 2090 & $512 \pi h \times 2 h \times 8 \pi h$ & $16384 \times 128 \times 512$ \\
\hline \hline
\end{tabular}

number is fixed at $R e_{\tau}=u_{\tau} h / v=180$. The values of the corresponding mean-velocity-based rotation number $R o_{c}=2 \Omega h / U_{c}$ and Reynolds number $R e_{c}=U_{c} h / v$ are also given in the table, where $U_{c}$ is the mean velocity at the channel center. The criteria for determining the computational domain size $L_{i}$ and number of grid points $N_{i}$ are discussed in detail in the work of Yang and Wang. ${ }^{18}$ In summary, as the rotation number increases, a larger streamwise domain size $L_{1}$ with a larger number of grid points $N_{1}$ is needed to capture the streamwise-elongated vortex structures, of which the characteristic streamwise scale extends as the rotation number increases. The longest computational domain used in the DNS is $L_{1}=512 \pi h$ for the case of $R o_{\tau}=150$. In contrast, the characteristic spanwise scale of the large-scale vortices remains almost unchanged at various rotation numbers such that $L_{3}=8 \pi h$ with $N_{3}=512$ is fixed in all cases. In our previous studies, the DNS data were analyzed to investigate the effect of streamwise system rotation on the pressure field ${ }^{19}$ and the sustaining mechanism of large-scale vortices. ${ }^{17}$ In the present study, we focus on investigating the non-monotonic dynamic behavior of the magnitude of the cross-stream secondary flow in response to the increase in the rotation number.

\section{EFFECT OF ROTATION NUMBER ON MEAN SPANWISE VELOCITY}

Figure 2 compares the profiles of the mean spanwise velocity $\left\langle u_{3}\right\rangle^{+}$at six rotation numbers. In this paper, the superscript "+" denotes variables non-dimensionalized using $v / u_{\tau}$ and $u_{\tau}$ as characteristic length and velocity scales, respectively. Given that the profiles of $\left\langle u_{3}\right\rangle^{+}$are antisymmetric about the central plane $\left(x_{2} / h=0\right)$ of the channel, they are only displayed in the lower half of the channel (for $\left.-1 \leq x_{2} / h \leq 0\right)$. From the inset graph, which shows the profile of $\left\langle u_{3}\right\rangle^{+}$at $R o_{\tau}=150$ across the entire channel, it is seen that the sign of $\left\langle u_{3}\right\rangle^{+}$changes three times between two channel walls, resulting in four distinct layers of opposite motions. The magnitude of the near-wall negatively valued peak of $\left\langle u_{3}\right\rangle^{+}$(located at $x_{2} / h=-0.96$ to -0.89$)$ reaches its maximum at $R o_{\tau}=30$. The magnitude-reverse phenomenon of $\left\langle u_{3}\right\rangle^{+}$occurring at $R o_{\tau} \approx 30$ observed in Fig. 2 is consistent with the findings of Weller and Oberlack ${ }^{4}$ and Yang, $\mathrm{Su}$, and $\mathrm{Wu},{ }^{11}$ who reported that the magnitude of $\left\langle u_{3}\right\rangle^{+}$reverses around $R o_{\tau}$ $=14-30$ at similar Reynolds numbers.

To further investigate the non-monotonic behavior of the mean spanwise velocity, we perform time and plane averaging on the third-component of Eq. (2) and integrate the resultant equation in the $x_{2}$-direction, leading to the following balance equation of the 


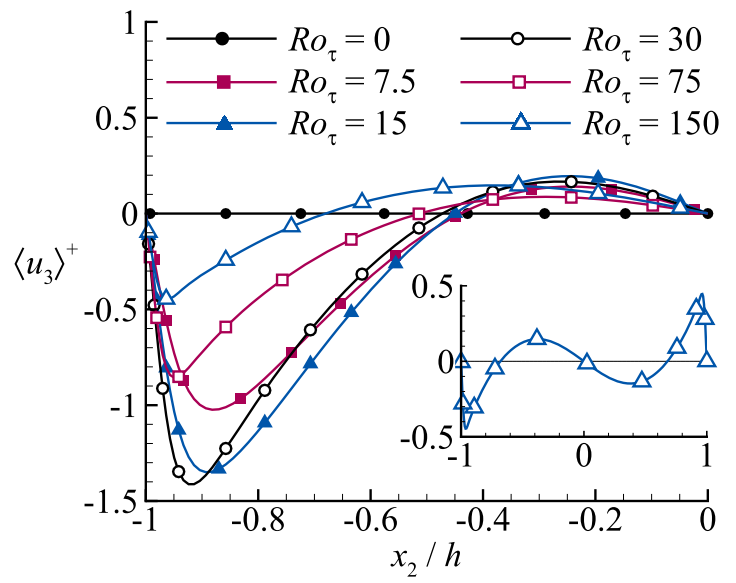

FIG. 2. Profiles of mean spanwise velocity $\left\langle u_{3}\right\rangle^{+}$at various rotation numbers. In order to demonstrate the structures of secondary mean flow, the profile of mean spanwise velocity $\left\langle u_{3}\right\rangle^{+}$at $R o_{\tau}=150$ is also displayed across the entire channel $\left(-1 \leq x_{2} / h \leq 1\right)$ in an inset graph, which shows a characteristic pattern of four distinct layers of opposite motions.

spanwise shear stress:

$$
\frac{\mathrm{d}\left\langle u_{3}\right\rangle^{+}}{\mathrm{d} x_{2}^{+}}=\left\langle u_{2}^{\prime} u_{3}^{\prime}\right\rangle^{+}+\tau_{23}^{\text {wall }+},
$$

where $\tau_{23}^{\text {wall }+}=\left(\mathrm{d}\left\langle u_{3}\right\rangle^{+} / \mathrm{d} x_{2}^{+}\right)_{x_{2}^{+}=-h^{+}}$represents the spanwise wall shear stress. Equation (3) shows a linear relationship between $\mathrm{d}\left\langle u_{3}\right\rangle^{+} / \mathrm{d} x_{2}^{+}$and $\left\langle u_{2}^{\prime} u_{3}^{\prime}\right\rangle^{+}$, suggesting that it would be helpful to examine the profiles of $\left\langle u_{2}^{\prime} u_{3}^{\prime}\right\rangle^{+}$to understand the non-monotonic behavior of the magnitude of $\left\langle u_{3}\right\rangle^{+}$.

Figure 3 compares the profiles of Reynolds stresses $\left\langle u_{2}^{\prime} u_{3}^{\prime}\right\rangle^{+}$at six rotation numbers. As expected, the variation in the peak value of $\left\langle u_{2}^{\prime} u_{3}^{\prime}\right\rangle^{+}$is also non-monotonic with respect to an increasing rotation number. The magnitude of $\left\langle u_{2}^{\prime} u_{3}^{\prime}\right\rangle^{+}$is the largest at $R o_{\tau}=30$ among all cases under investigation. The reverse effects of the rotation number on the magnitudes of $\left\langle u_{3}\right\rangle^{+}$and $\left\langle u_{2}^{\prime} u_{3}^{\prime}\right\rangle^{+}$are intriguing.

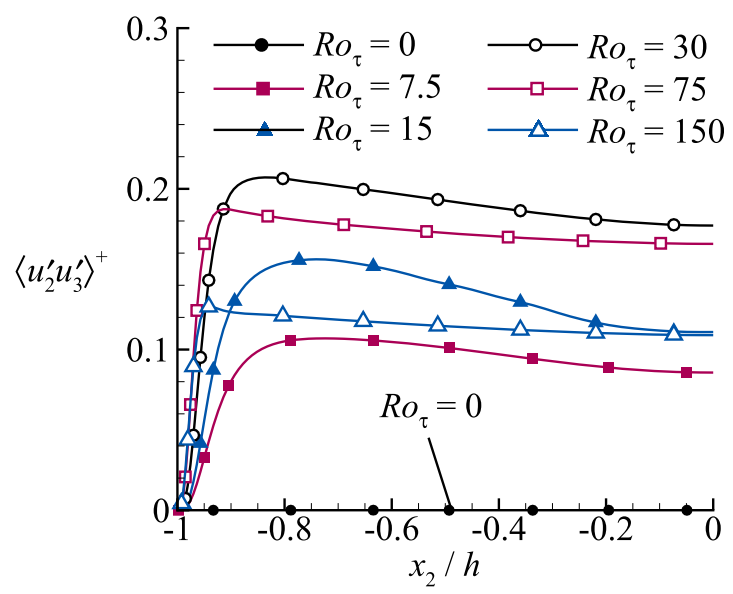

FIG. 3. Profiles of Reynolds stresses $\left\langle u_{2}^{\prime} u_{3}^{\prime}\right\rangle^{+}$at various rotation numbers.
In Sec. IV, these phenomena are further investigated by seeking their physical explanations through the budget analysis of the Reynolds shear stress $\left\langle u_{2}^{\prime} u_{3}^{\prime}\right\rangle^{+}$.

\section{SELF-CONSTRAINT MECHANISM OF REYNOLDS SHEAR STRESS $\left\langle u_{2}^{\prime} u_{3}^{\prime}\right\rangle^{+}$}

In this section, the non-monotonic behaviors of $\left\langle u_{3}\right\rangle^{+}$and $\left\langle u_{2}^{\prime} u_{3}^{\prime}\right\rangle^{+}$are further investigated through the analysis of the transport equation of the Reynolds stress $\left\langle u_{i}{ }^{\prime} u_{j}^{\prime}\right\rangle$, expressed as

$$
\frac{\partial\left\langle u_{i}^{\prime} u_{j}^{\prime}\right\rangle}{\partial t}=0=P_{i j}+C_{i j}^{\mathrm{eff}}+\Pi_{i j}^{c}+\varepsilon_{i j}+T_{i j}+D_{i j} .
$$

Here, $P_{i j}, C_{i j}^{\text {eff }}, \Pi_{i j}^{c}, \varepsilon_{i j}, T_{i j}$, and $D_{i j}$ denote the production term, effective rotation term, convection-induced pressure term, dissipation term, turbulent diffusion term, and viscous diffusion term, respectively, defined as

$$
\begin{gathered}
P_{i j}=-\left(\left\langle u_{i}^{\prime} u_{k}^{\prime}\right\rangle \frac{\partial\left\langle u_{j}\right\rangle}{\partial x_{k}}+\left\langle u_{j}^{\prime} u_{k}^{\prime}\right\rangle \frac{\partial\left\langle u_{i}\right\rangle}{\partial x_{k}}\right), \\
C_{i j}^{\mathrm{eff}}=2 \Omega\left(\varepsilon_{1 i k}\left\langle u_{j}^{\prime} u_{k}^{\prime}\right\rangle+\varepsilon_{1 j k}\left\langle u_{i}^{\prime} u_{k}^{\prime}\right\rangle\right) \\
-\frac{1}{\rho}\left\langle u_{i}^{\prime} \frac{\partial p_{r}^{\prime}}{\partial x_{j}}+u_{j}^{\prime} \frac{\partial p_{r}^{\prime}}{\partial x_{i}}\right\rangle, \\
\Pi_{i j}^{c}=-\frac{1}{\rho}\left\langle u_{i}^{\prime} \frac{\partial p_{c}^{\prime}}{\partial x_{j}}+u_{j}^{\prime} \frac{\partial p_{c}^{\prime}}{\partial x_{i}}\right\rangle, \\
\varepsilon_{i j}=-2 v\left\langle\frac{\partial u_{i}^{\prime}}{\partial x_{k}} \frac{\partial u_{j}^{\prime}}{\partial x_{k}}\right\rangle, \\
T_{i j}=-\frac{\partial\left\langle u_{i}^{\prime} u_{j}^{\prime} u_{k}^{\prime}\right\rangle}{\partial x_{k}}, \\
D_{i j}=v \frac{\partial^{2}\left\langle u_{i}^{\prime} u_{j}^{\prime}\right\rangle}{\partial x_{k} \partial x_{k}} .
\end{gathered}
$$

In Eq. (6), the effective rotation term consists of two parts, namely, the Coriolis term $C_{i j}$ and the rotation-induced pressure term $\Pi_{i j}^{r}$, which are defined, respectively, as

$$
\begin{gathered}
C_{i j}=2 \Omega\left(\varepsilon_{1 i k}\left\langle u_{j}^{\prime} u_{k}^{\prime}\right\rangle+\varepsilon_{1 j k}\left\langle u_{i}^{\prime} u_{k}^{\prime}\right\rangle\right), \\
\Pi_{i j}^{r}=-\frac{1}{\rho}\left\langle u_{i}^{\prime} \frac{\partial p_{r}^{\prime}}{\partial x_{j}}+u_{j}^{\prime} \frac{\partial p_{r}^{\prime}}{\partial x_{i}}\right\rangle .
\end{gathered}
$$

The Coriolis term $C_{i j}$, as noted in many previous studies of rotating flows, ${ }^{18,20-24}$ represents the effect of the system rotation on the transport equations of Reynolds stresses. The rotation-induced pressure term, which is usually absorbed into the pressure term in previous studies, should be also regarded as a direct consequence caused by the imposed system rotation. This point can be explained by the definition of the rotation-induced pressure given below.

In Eqs. (7) and (12), the pressure $p$ is decomposed into a convection-induced part $p^{c}$ and a rotation-induced part $p^{r}$. The convection-induced pressure $p^{c}$ is governed by the following Poisson equation and boundary conditions:

$$
\left\{\begin{array}{cl} 
& \frac{1}{\rho} \frac{\partial^{2} p_{c}}{\partial x_{i} \partial x_{i}}=-\frac{\partial u_{i}}{\partial x_{j}} \frac{\partial u_{j}}{\partial x_{i}}, \\
\text { with } & \frac{\partial p_{c}}{\partial x_{2}}=\rho v \frac{\partial^{2} u_{2}}{\partial x_{2}^{2}} \quad \text { at } \quad x_{2}= \pm h
\end{array}\right.
$$


while the Poisson equation and boundary condition for the rotationinduced pressure $p_{r}$ are expressed as

$$
\left\{\begin{array}{c}
\frac{1}{\rho} \frac{\partial^{2} p_{r}}{\partial x_{i} \partial x_{i}}=2 \Omega \omega_{1}, \\
\text { with } \quad \frac{\partial p_{r}}{\partial x_{2}}=0 \quad \text { at } \quad x_{2}= \pm h .
\end{array}\right.
$$

The above decomposition of pressure was proposed in our previous study of the effect of system rotation on the pressure field. ${ }^{1}$ This method for pressure decomposition is not only rigorous in mathematics but also conceptually clear in flow physics by separating the non-rotating and rotating effects on the pressure field. The rotation-induced pressure $p_{r}$ is a direct consequence of the imposed streamwise system rotation, and therefore, the Coriolis term $C_{i j}$ and the rotation-induced pressure term $\Pi_{i j}^{r}$ are combined as an effective rotation term $C_{i j}^{\text {eff }}$ in Eq. (6) for the analysis of the budget of Reynolds stresses. In our previous study of the sustaining mechanism of Taylor-Görtler-like vortices, ${ }^{17}$ similar transport equations of energy spectra were investigated. A similar definition of the effective rotation term was found to be more appropriate than either the Coriolis term or rotation-induced pressure term for representing the effect of the streamwise system rotation on the flow dynamics. Different from our previous study, which focuses on the normal components of energy spectra, the present study aims to investigate the reversal behavior of Reynolds shear stress $\left\langle u_{2}^{\prime} u_{3}^{\prime}\right\rangle$ by analyzing its budget balance in the physical space.
Figure 4 compares the profiles of budget terms in the transport equation of Reynolds shear stress $\left\langle u_{2}^{\prime} u_{3}^{\prime}\right\rangle^{+}$at $R o_{\tau}=7.5$ (the lowest rotation number for the rotating channel flow under investigation), $R o_{\tau}=30$ (the critical rotation number at which the magnitude of $\left\langle u_{2}^{\prime} u_{3}^{\prime}\right\rangle^{+}$reverses, see Fig. 3), and $R o_{\tau}=150$ (the highest rotation number under investigation). To ensure that the flow is developed to a statistically stationary state, it is useful to examine the residual of the summation of all budget terms in Eq. (4), which is found to be smaller than $1 \%$ of the dominant source terms in all cases under investigation. Furthermore, the time averaging in the present study is conducted over a time duration of $T=50 \mathrm{~h} / \mathrm{u}_{\tau}$. We have also calculated the budget terms by reducing the averaging time duration to $T$ $=25 h / u_{\tau}$, which does not cause any increase in the residual magnitude. This indicates that the averaging time is sufficiently long, and the error is mainly attributed to the spatial discretization, but not the assumption that the flow is statistically stationary. From Fig. 4(a), it is clear that the convection-induced pressure term $\Pi_{23}^{c+}$ and effective rotation term $C_{23}^{\text {eff+ }}$ are two dominant sources of $\left\langle u_{2}^{\prime} u_{3}^{\prime}\right\rangle^{+}$at $R o_{\tau}=7.5$. At all three rotation numbers, the convection-induced pressure term $\Pi_{23}^{c+}$ acts as an important source, and its effect becomes increasingly dominant as the rotation number increases. Furthermore, by comparing Figs. 4(a)-4(c), it is seen that the effect of $C_{23}^{\text {eff }+}$ (as a gain for $\left\langle u_{2}^{\prime} u_{3}^{\prime}\right\rangle^{+}$) is the most apparent at $R o_{\tau}=30$.

To further investigate the effective rotation term $C_{23}^{\text {eff+ }}$, we compare its profiles at various rotation numbers, together with the Coriolis term $C_{23}^{+}$[Eq. (11)] and rotation-induced pressure term $\Pi_{23}^{r+}$ [Eq. (12)] in Fig. 5. It is seen from Fig. 5(b) that the Coriolis term $C_{23}^{+}$
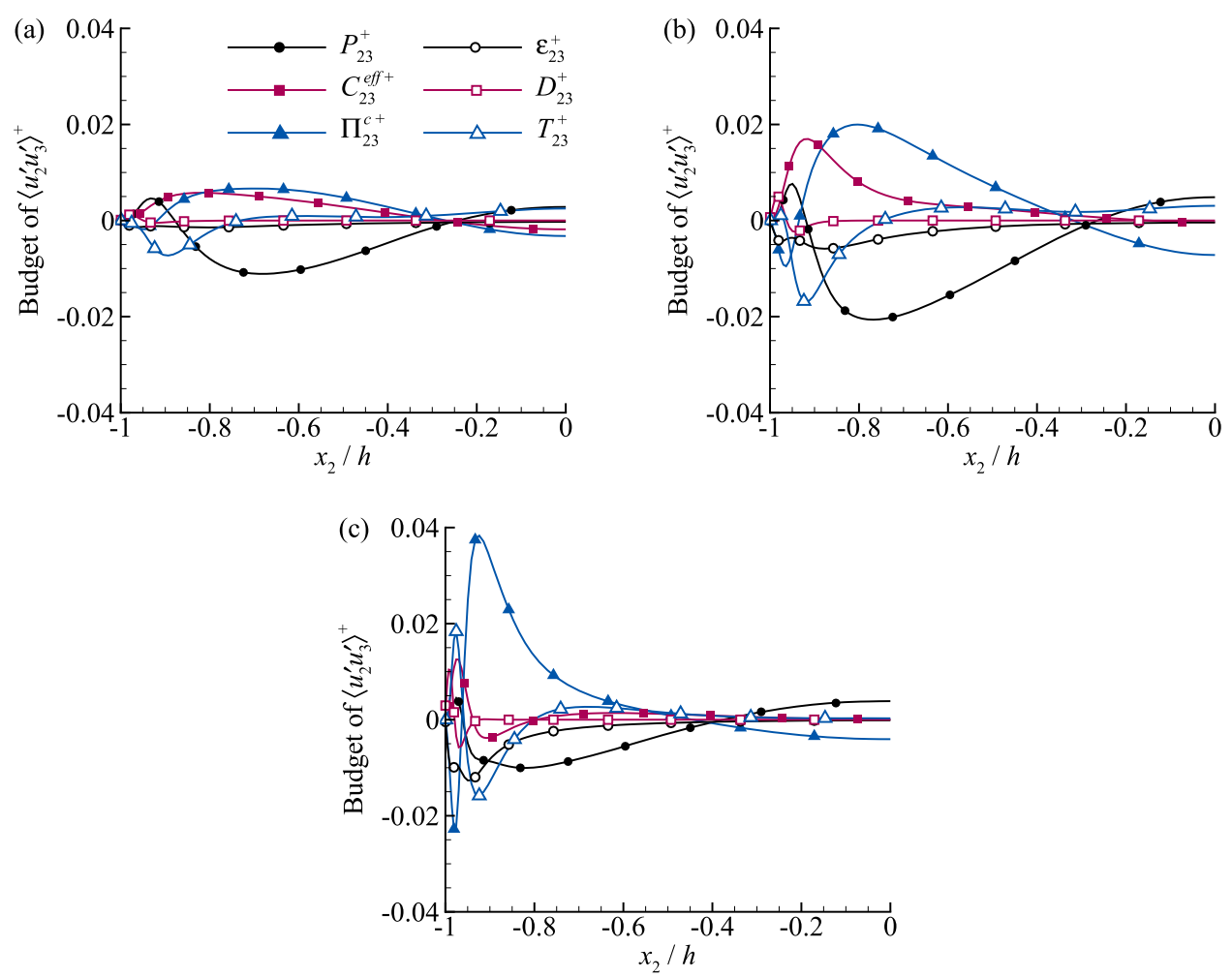

FIG. 4. Profiles of budget terms in the transport equation of Reynolds shear stress $\left\langle u_{2}^{\prime} u_{3}^{\prime}\right\rangle^{+}$in the streamwiserotating turbulent channel flow at (a) $R o_{\tau}$ $=7.5$, (b) $R o_{\tau}=30$, and (c) $R o_{\tau}=150$. 


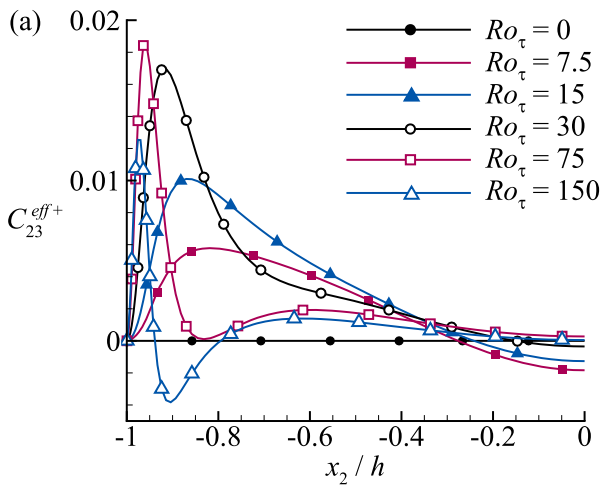

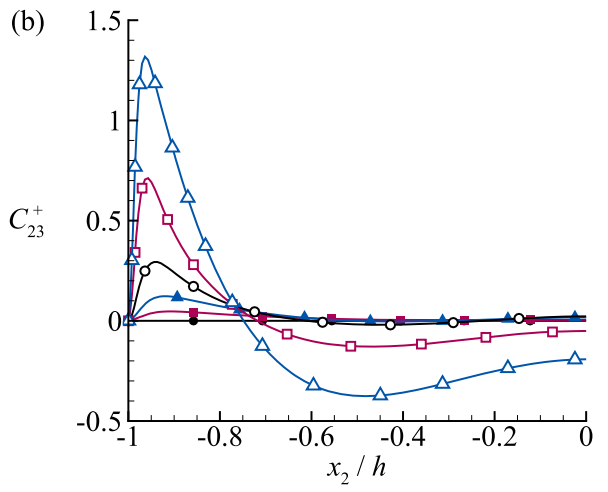

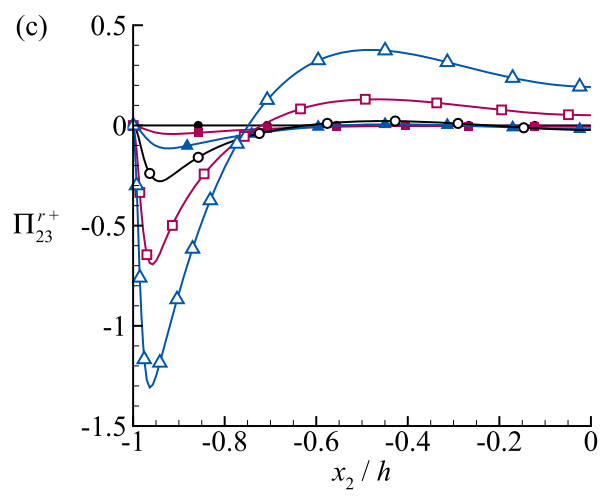

FIG. 5. Profiles of (a) effective rotation term $C_{23}^{\text {eff+ }}$, (b) Coriolis term $C_{i j}^{+}$, and (c) rotation-induced pressure term $\Pi_{i j}^{r+}$ at various rotation numbers. is positively valued in the near-wall region, while in the core region of the channel, the value of $C_{23}^{+}$is negative at high rotation numbers $\left(R o_{\tau}=75\right.$ and 150). The sign of $C_{23}^{+}$can be understood from its definition, expressed as

$$
C_{23}^{+}=R o_{\tau}\left(\left\langle u_{3}^{\prime} u_{3}^{\prime}\right\rangle^{+}-\left\langle u_{2}^{\prime} u_{2}^{\prime}\right\rangle^{+}\right)
$$

In the near wall region, due to the restriction of the wall, the magnitude of $\left\langle u_{2}^{\prime} u_{2}^{\prime}\right\rangle^{+}$is smaller than that of $\left\langle u_{3}^{\prime} u_{3}^{\prime}\right\rangle^{+}$, leading to the positive value of $C_{23}^{+}$. At high rotation numbers, the occurrence of intensive large-scale Taylor-Görtler-like vortices enhances the wall-normal velocity fluctuation $u_{2}{ }^{\prime}$ in the core region of the channel, ${ }^{17}$ and consequently, the sign of $C_{23}^{+}$becomes negative there. The rotationinduced pressure term $\Pi_{23}^{r+}$ depicted in Fig. 5(c) almost form a mirror image of $C_{23}^{+}$at all rotation numbers under investigation. This indicates that these two rotation-induced terms $C_{23}^{+}$and $\Pi_{23}^{r+}$ mostly cancel the effect of each other, leaving a significantly smaller net effect of the imposed system rotation on the budget balance of $\left\langle u_{2}^{\prime} u_{3}^{\prime}\right\rangle^{+}$. As a result, although the magnitudes of both $C_{i j}^{+}$and $\Pi_{i j}^{r+}$ increase approximately in a linear approach with respect to the rotation number (a phenomenon that can be understood from the definitions of $C_{i j}^{+}$and $\left.\Pi_{i j}^{r+}\right)$, the net effect of the system rotation on $\left\langle u_{2}^{\prime} u_{3}^{\prime}\right\rangle^{+}$as represented by the effective rotation term $C_{23}^{\text {eff+ }}$ is non-monotonic. As shown in Fig. 5(a), the peak value of $C_{23}^{\text {eff }}$ reaches its maximum at $R o_{\tau}=75$. This rotation number is higher than the critical reversal rotation number of $\left\langle u_{2}^{\prime} u_{3}^{\prime}\right\rangle^{+}\left(R o_{\tau}=30\right.$ as shown in Fig. 3$)$, indicating that the effect of $C_{23}^{\text {eff+ }}$ is important, but meanwhile not primary, in the non-monotonically behavior of $\left\langle u_{2}^{\prime} u_{3}^{\prime}\right\rangle^{+}$.
Another term that plays a crucial role in the magnitude-reverse phenomenon of $\left\langle u_{2}^{\prime} u_{3}^{\prime}\right\rangle^{+}$is the production term $P_{23}^{+}$. As shown in Fig. 4, the profiles of the production term $P_{23}^{+}$are complex. From Eq. (5), we obtain

$$
P_{23}^{+}=-\left\langle u_{2}^{\prime} u_{2}^{\prime}\right\rangle^{+} \frac{\mathrm{d}\left\langle u_{3}\right\rangle^{+}}{\mathrm{d} x_{2}^{+}}
$$

from which it can be inferred that the profile of $P_{23}^{+}$must cross zero six times in the wall-normal direction of the channel (for $-1 \leq x_{2} / h$ $\leq 1$ ) or, equivalently, three times in the lower half of the channel (for $\left.-1 \leq x_{2} / h \leq 0\right)$, as shown in Fig. 4. Owing to the wall restriction, $P_{23}^{+}$ $=0$ holds at the two walls (i.e., at $x_{2} / h= \pm 1$ ). Furthermore, as shown in Fig. 2, there are four layers of opposite mean spanwise motion $\left\langle u_{3}\right\rangle^{+}$. In each layer, there exists a wall-normal position $x_{2}$ where the magnitude of the spanwise velocity $\left\langle u_{3}\right\rangle^{+}$reaches its local maximum, where $\mathrm{d}\left\langle u_{3}\right\rangle^{+} / \mathrm{d} x_{2}^{+}=0$ holds. Therefore, the other four zero points of $P_{23}^{+}$collocate with the four peaks of $\left\langle u_{3}\right\rangle^{+}$. In summary, given the four distinct layers of the opposite mean spanwise motion, the sign of $P_{23}^{+}$must change four times in the entire channel.

It is seen from Fig. 4 that the value of $P_{23}^{+}$is mostly negative across the wall-normal direction (e.g., at $R o_{\tau}=30, P_{23}^{+}<0$ holds in the region for $\left.-0.92 \leq x_{2} / h \leq-0.27\right)$. This indicates that $P_{23}^{+}$serves as a sink in the transport equation of $\left\langle u_{2}^{\prime} u_{3}^{\prime}\right\rangle^{+}$, which tends to suppress the magnitude of this Reynolds shear stress component. By comparing Figs. 4(a)-4(c), it is clear that the magnitude of the negatively valued peak of $P_{23}^{+}$is the largest at $R o_{\tau}=30$ among the three rotation numbers. To demonstrate this trend more clearly, the profiles of $P_{23}^{+}$ at six rotation numbers are compared in Fig. 6. Similar to $\left\langle u_{2}^{\prime} u_{3}^{\prime}\right\rangle^{+}$, the magnitude of the negatively valued peak of $P_{23}^{+}$also reverses at 


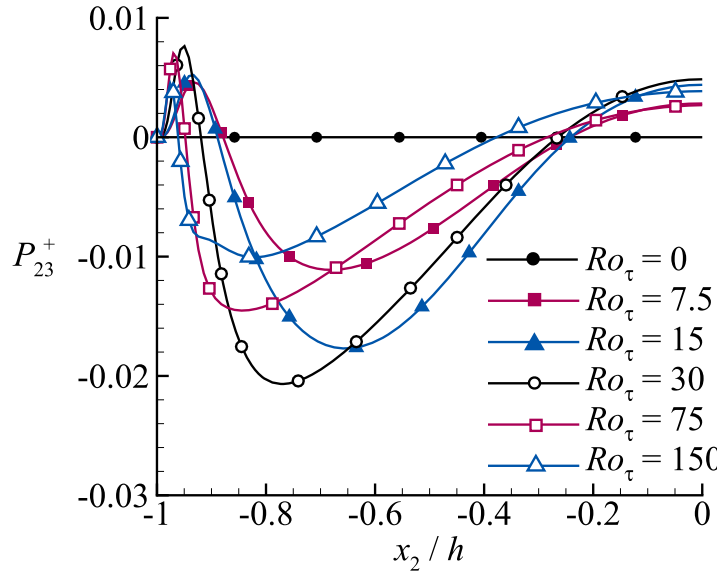

FIG. 6. Profiles of the production term $P_{23}^{+}$at various rotation numbers.

$R o_{\tau}=30$. From Eq. (5), we obtain

$$
P_{23}^{+}=-\left\langle u_{2}^{\prime} u_{2}^{\prime}\right\rangle^{+} \frac{\mathrm{d}\left\langle u_{3}\right\rangle^{+}}{\mathrm{d} x_{2}^{+}} .
$$

Substituting Eq. (3) into (17) results in

$$
P_{23}^{+}=-\left\langle u_{2}^{\prime} u_{2}^{\prime}\right\rangle^{+}\left(\left\langle u_{2}^{\prime} u_{3}^{\prime}\right\rangle^{+}+\tau_{23}^{\text {wall }+}\right) .
$$

Given the fact that $\left\langle u_{2}^{\prime} u_{2}^{\prime}\right\rangle^{+}$is positive semi-definite, Eq. (18) indicates that $\left\langle u_{2}^{\prime} u_{3}^{\prime}\right\rangle^{+}$always imposes a negative net feedback to itself through its production term $P_{23}^{+}$. To be specific, as the rotation number increases from 0 to 30 , accompanied with the magnitude increase in $\left\langle u_{2}^{\prime} u_{3}^{\prime}\right\rangle^{+}$, the suppression effect of the production term $P_{23}^{+}$becomes stronger. This "self-constraint" mechanism prevents the magnitude of $\left\langle u_{2}^{\prime} u_{3}^{\prime}\right\rangle^{+}$from monotonically increasing as the rotation number continues to increase, and as a result, the magnitude of $\left\langle u_{2}^{\prime} u_{3}^{\prime}\right\rangle^{+}$shows a non-monotonic behavior in Fig. 3 .

The magnitude reverse phenomenon of the near-wall peak of $\left\langle u_{3}\right\rangle^{+}$with respect to an increasing rotation number is coupled with that of $\left\langle u_{2}^{\prime} u_{3}^{\prime}\right\rangle^{+}$. To demonstrate it, we integrate Eq. (3) from $-h^{+}$ to $h^{+}$. Further considering the no-slip condition at the two walls and the symmetry in the profile of $\left\langle u_{2}^{\prime} u_{3}^{\prime}\right\rangle^{+}$about the channel center $\left(x_{2} / h\right.$ $=0$ ), we obtain

$$
\tau_{23}^{\text {wall }+}=-\frac{1}{h^{+}} \int_{-h^{+}}^{0}\left\langle u_{2}^{\prime} u_{3}^{\prime}\right\rangle^{+} \mathrm{d} x_{2}^{+} .
$$

From Eq. (19), it is understood that the value of $\tau_{23}^{\text {wall }+}$ is proportional to the integration of $\left\langle u_{2}^{\prime} u_{3}^{\prime}\right\rangle^{+}$over the lower half of the channel. Figure 3 shows that the magnitude of $\left\langle u_{2}^{\prime} u_{3}^{\prime}\right\rangle^{+}$reverses at $R o_{\tau}=30$, and consequently, $\tau_{23}^{\text {wall }+}$ also reverses at $R o_{\tau}=30$. In the near-wall region, the mean spanwise velocity can be expanded into Taylor series as

$$
\left\langle u_{3}\right\rangle^{+}=\left.\frac{\mathrm{d}\left\langle u_{3}\right\rangle^{+}}{\mathrm{d} x_{2}^{+}}\right|_{x_{2}^{+}=-h^{+}} y^{+}+\mathscr{O}\left(y^{+2}\right)=\tau_{23}^{\text {wall }+} y^{+}+\mathscr{O}\left(y^{+2}\right),
$$

indicating that the behaviors of the near-wall peak of $\left\langle u_{3}\right\rangle^{+}$and $\tau_{23}^{\text {wall }+}$ must synchronize in response to an increasing rotation number. As a result, the near-wall peak of $\left\langle u_{3}\right\rangle^{+}$also reverses at the same rotation number (see Fig. 2).

\section{CONCLUSIONS}

In this paper, we investigate the mechanism underlying the non-monotonic behavior of the cross-stream secondary flow $\left\langle u_{3}\right\rangle$ with respect to an increasing rotation number in the streamwiserotating turbulent channel flow. Using the DNS data, it is demonstrated that the magnitudes of both $\left\langle u_{3}\right\rangle$ and $\left\langle u_{2}^{\prime} u_{3}^{\prime}\right\rangle$ reverse at $R o_{\tau}$ $=30$. Through the analysis of the transport equation of $\left\langle u_{2}^{\prime} u_{3}^{\prime}\right\rangle$, it is found that $\left\langle u_{2}^{\prime} u_{3}^{\prime}\right\rangle$ imposes a negative feedback to itself through the production term $P_{23}$. This self-contraint mechanism prevents the magnitude of $\left\langle u_{2}^{\prime} u_{3}^{\prime}\right\rangle$ from increasing monotonically in response to an increasing rotation number. The effective rotation term plays a secondary role in the magnitude reverse of $\left\langle u_{2}^{\prime} u_{3}^{\prime}\right\rangle$. The two components of the effective rotation term, namely, the Coriolis term and rotation-induced pressure term, both increase approximately in a linear approach with respect to the rotation number. However, their effects mostly cancel each other, leaving a significantly smaller net effect on the budget balance of $\left\langle u_{2}^{\prime} u_{3}^{\prime}\right\rangle$ as represented by the effective rotation term. The peak value of the effective rotation term reverses at $R o_{\tau}=75$, larger than the critical reversal rotation number of $\left\langle u_{2}^{\prime} u_{3}^{\prime}\right\rangle$. Due to the linear relationship between the spanwise wall shear stress $\tau_{23}^{\text {wall }}$ and the integration of $\left\langle u_{2}^{\prime} u_{3}^{\prime}\right\rangle$, the value of $\tau_{23}^{\text {wall }}$ also reverses at $R o_{\tau}=30$. Furthermore, through a Taylor-series analysis, it is shown that the near-wall behavior of $\left\langle u_{3}\right\rangle$ depends linearly on that of $\tau_{23}^{\text {wall }}$. As such, the magnitude-reverse phenomenon of $\left\langle u_{3}\right\rangle$ is explained.

\section{ACKNOWLEDGMENTS}

Z.Y. would like to thank the support of the NSFC Basic Science Center Program for "Multiscale Problems in Nonlinear Mechanics" (Grant No. 11988102) and the Lixing research funding of Institute of Mechanics, Chinese Academy of Sciences. The DNS database was established under the support of the Natural Sciences and Engineering Research Council (NSERC) of Canada to B.-C.W.

\section{DATA AVAILABILITY}

Raw data were generated at the Western Canada Research Grid (WestGrid) large scale facility. The data that support the findings of this study are available from the corresponding author upon reasonable request.

\section{REFERENCES}

${ }^{1} \mathrm{H}$. Wu and N. Kasagi, "Effects of arbitrary directional system rotation on turbulent channel flow,” Phys. Fluids 16, 979-990 (2004).

${ }^{2}$ M. Oberlack, W. Cabot, B. A. P. Reif, and T. Weller, "Group analysis, direct numerical simulation and modelling of a turbulent channel flow with streamwise rotation," J. Fluid Mech. 562, 383-403 (2006).

${ }^{3}$ T. Weller and M. Oberlack, "DNS of a turbulent channel flow with streamwise rotation-Investigation on the cross flow phenomena," in Direct and Large-Eddy Simulation VI, edited by E. Lamballais, R. Friedrich, B. J. Geurts, and O. Métais (Springer, 2006), pp. 241-248.

${ }^{4}$ T. Weller and M. Oberlack, "DNS of a turbulent channel flow with streamwise rotation-Study of the reverse effect of the cross flow," Proc. Appl. Math. Mech. 6, 553-554 (2006).

${ }^{5}$ I. Recktenwald, T. Weller, W. Schröder, and M. Oberlack, "Comparison of direct numerical simulations and particle-image velocimetry data of turbulent channel flow rotating about the streamwise axis," Phys. Fluids 19, 085114 (2007). 
${ }^{6}$ N. Alkishriwi, M. Meinke, and W. Schröder, "Large-eddy simulation of streamwise-rotating turbulent channel flow," Comput. Fluids 37, 786-792 (2008).

${ }^{7}$ I. Recktenwald, N. Alkishriwi, and W. Schröder, "PIV-LES analysis of channel flow rotating about the streamwise axis," Eur. J. Mech.: B/Fluids 28, 677-688 (2009).

${ }^{8}$ B.-C. Wang and Y. Zhang, "Large-eddy simulation of turbulent flows in a heated streamwise rotating channel," Int. J. Heat Fluid Flow 44, 71-86 (2013).

${ }^{9}$ Y.-J. Dai, W.-X. Huang, and C.-X. Xu, "Coherent structures in streamwise rotating channel flow," Phys. Fluids 31, 021204 (2019).

${ }^{10}$ S. Masuda, S. Fukuda, and M. Nagata, "Instabilities of plane Poiseuille flow with a streamwise system rotation," J. Fluid Mech. 603, 189-206 (2008).

${ }^{11}$ Y.-T. Yang, W.-D. Su, and J.-Z. Wu, "Helical-wave decomposition and applications to channel turbulence with streamwise rotation," J. Fluid Mech. 662, 91-122 (2010).

${ }^{12}$ N. N. Mansour, J. Kim, and P. Moin, "Reynolds-stress and dissipation-rate budgets in a turbulent channel flow," J. Fluid Mech. 194, 15-44 (1988).

${ }^{13}$ J. Komminaho and M. Skote, "Reynolds stress budgets in Couette and boundary layer flows," Flow, Turbul. Combust. 68, 167-192 (2002).

${ }^{14} \mathrm{~S}$. Hoyas and J. Jiménez, "Reynolds number effects on the Reynolds-stress budgets in turbulent channels," Phys. Fluids 20, 101511 (2008).

${ }^{15}$ V. Avsarkisov, S. Hoyas, M. Oberlack, and J. P. García-Galache, "Turbulent plane Couette flow at moderately high Reynolds number," J. Fluid Mech. 751, R1 (2014).
${ }^{16}$ T. Kawata and P. H. Alfredsson, "Scale interactions in turbulent rotating planar Couette flow: Insight through the Reynolds stress transport," J. Fluid Mech. 879, 255-295 (2019).

${ }^{17}$ Z. Yang, B.-Q. Deng, B.-C. Wang, and L. Shen, "Sustaining mechanism of Taylor-Görtler-like vortices in a streamwise-rotating channel flow," Phys. Rev. Fluids 5, 044601 (2020).

${ }^{18} \mathrm{Z}$. Yang and B.-C. Wang, "Capturing Taylor-Görtler vortices in a streamwiserotating channel at very high rotation numbers," J. Fluid Mech. 838, 658-689 (2018).

${ }^{19}$ Z. Yang, B.-Q. Deng, B.-C. Wang, and L. Shen, "Letter: The effects of streamwise system rotation on pressure fluctuations in a turbulent channel flow," Phys. Fluids 30, 091701 (2018).

${ }^{20} \mathrm{~K}$. Nakabayashi and O. Kitoh, "Turbulence characteristics of two-dimensional channel flow with system rotation," J. Fluid Mech. 528, 355-377 (2005).

${ }^{21}$ N.-S. Liu and X.-Y. Lu, "A numerical investigation of turbulent flows in a spanwise rotating channel," Comput. Fluids 36, 282-298 (2007).

${ }^{22}$ O. Grundestam, S. Wallin, and A. V. Johansson, "Direct numerical simulations of rotating turbulent channel flow," J. Fluid Mech. 598, 177-199 (2008).

${ }^{23}$ T. Kawata and P. H. Alfredsson, "Experiments in rotating plane Couette flowMomentum transport by coherent roll-cell structure and zero-absolute-vorticity state," J. Fluid Mech. 791, 191-213 (2016).

${ }^{24}$ T. Kawata and P. H. Alfredsson, "Turbulent rotating plane Couette flow: Reynolds and rotation number dependency of flow structure and momentum transport," Phys. Rev. Fluids 1, 034402 (2016). 